\title{
Fault identification and analysis of complex electromechanical system using dynamic fault probability
}

\author{
Hongxia $\mathrm{Wu}^{1, \mathrm{a}}$, Jie $\mathrm{Han}^{2, \mathrm{~b}}$, Dongchen Qin ${ }^{3, \mathrm{c}}$ \\ ${ }^{1}$ School of Mechanical Engineering, Zhengzhou University, Zhengzhou 450001, China \\ ${ }^{1}$ School of Chemical Engineering and Energy, Zhengzhou University, Zhengzhou 450001, China \\ ${ }^{2}$ School of Mechanical Engineering, Zhengzhou University, Zhengzhou 450001, China \\ ${ }^{3}$ School of Mechanical Engineering, Zhengzhou University, Zhengzhou 450001, China \\ awuh_x@sina.com, ${ }^{\text {bhj_email@163.com, }{ }^{c} d c q @ z z u . e d u . c n}$
}

Keywords: dynamic Bayesian network, dynamic fault probability, complex electromechanical system, fault diagnosis.

Abstract. This paper presents a novel fault identification method based on object oriented Bayesian network (OOBN) and dynamic fault probability according to the structure-function relationship and the failure mode effect analysis (FMEA) of the diagnosed system. Fault identification of complex electromechanical system is difficult. The diagnostic model is in real time because it considers the change of fault probability depending on the working time and the system function state influenced by degradation of component reliability. The peculiarities of proposed approach are that the diagnosis model is hierarchical and dynamic, to depict actual character of nodes. Furthermore, the model is reusable and expandable. The application of ISG-engine in hybrid electric bus demonstrates the effectiveness and superiority of the approach.

\section{Introduction}

Energy diversification and the growing need for autonomous agents that control physical systems motivate the occurrence of complex hybrid systems. Larger-scale and more sophisticated system is more knowledge. Each of subsystems interrelates with each other. And what's more, the components function is degraded with working time. Fault probability always alters. We hope to detect the abnormal behavior and diagnose the failure for the system. Traditional fault diagnosis methods become difficult to complete this task. The intelligent diagnosis research has been focus on for this reason.

In recent years, some expert systems (Wang et al, 2010) and intelligent diagnosis methods based on model (David and Jose, 2000)had been made certain progress. Li (2006), Lerner (2000) and Bobbioa (2001) have shown that Bayesian Network is an useful classification for fault diagnosis since it explicates independencies between system components and diagnostic observations. Research on Bayesian network became especially popular. One of the critical issues in using Bayesian network as diagnostic tools is the construction of accurate and flexible diagnosis model. The majority of papers have been published related to creation of Bayesian network diagnosis model. Duan (2010) fused Bayesian Network and fault tree together for fault diagnosis to improve the diagnostic efficiency. Kawahara (2005) constructed a Dynamic Bayesian network for thrusters in maneuver of spacecraft using Kalman Filter Models(KFMs) and Hidden Markov Models(HMMs). However these methods don't give consideration to function states of systems and components, and moreover, they need effective algorithms to compute the transition matrix that defines the probabilities linking all states (David et al., 1999).

In this paper, we introduce an Object Oriented Approach and dynamic fault probability to define a dynamic fault diagnosis model. Firstly, we analyze structure-function and failure mode effect of 
system and construct BN for function object, then construct DBN for component object. Finally, the fault diagnosis model of ISG-engine based on OODBN is presented and the results are given.

\section{Bayesian Network For Function Object}

There are two technologies to construct fault diagnosis model based on OOBN. One is Top-Down Modeling (Bangso et al., 2000; Daphne et al., 1997). System fault is beginning node of BN. Then node objects are created and built into the BN. Another is Bottom-Up modeling (Weber et al., 2002). The BN model is designed by analyzing the systemic structure. After the system is decomposed and the sub-system objects and component objects are defined, the internal attributes of object are represented by means of BN. Sub-Bayesian Networks are connected according to relationship between sub-system and component. This method reflects the modular modeling idea.

In this paper, we fused the presented above two methods not only to improve diagnostic ability of model but also to help construction and maintenance of complex BN. The main technologies are constructing the BN by using both systemic function analysis and failure mode effect analysis (FMEA). Nodes are represented by function objects. Systemic function object lies in the highest level. Sub-function objects lie in middle levels. Component objects lie in the lowest level. Objects are connected according to relation of functional transmission. Failure modes are represented as attributes of function objects using random variables. The model is an OOBN representing whole function and states of system.

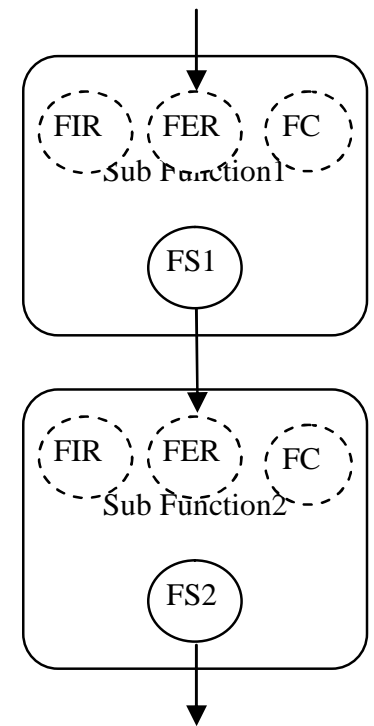

(a) Serial architecture

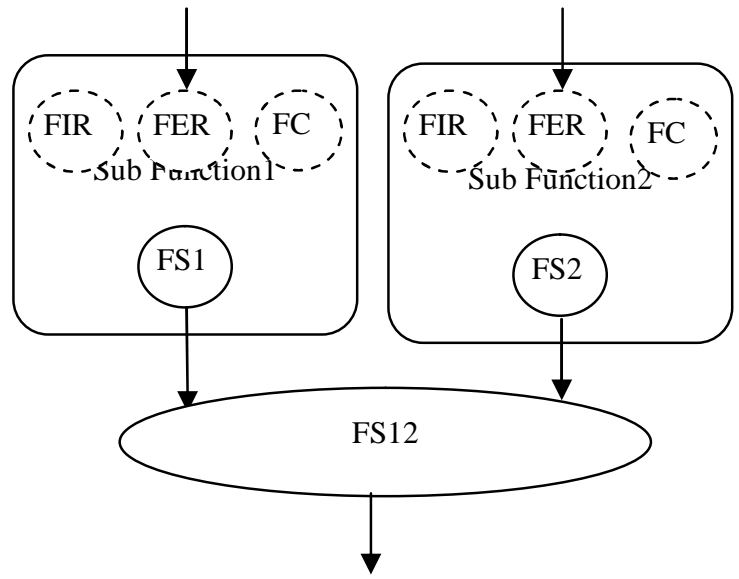

(b)Parallel architecture

Fig. (1). OOBN structure of function object

The structure of function object is shown in Fig.1, the relation between sub-functions is described in serial or parallel architectures. Functioning external resources(FER), functioning internal resources(FIR) and functioning constraints(FC) are input nodes. Functioning states (FS) information are output nodes.

In fig. (1a), the states information for sub-function 1 transmits sub-function 2 by input node FER. The states of sub-function1 affect the states of sub-function2. In fig.(1b), the states of two sub-functions are independent, the value of FS12 are done by the states of two subfunctions jointly. Relationship among function nodes are defined as conditional probability (CP). Components are linked with their function object nodes as input nodes. 


\section{Dynamic Bayesian Network For Component}

Yang has shown that DBN is a BN including a temporal dimension (Shahan et al.,2013). The fault probability of components and systems are all in a constant sate of flux with the longer service time. The service time of component is separated into slices. States information of component corresponding the time slices are described by sets of nodes $\left\{n_{0}, n_{1}, \ldots, n_{k}\right\}, n_{k-1}$ is parent node of $n_{k}$. Relationship between $n_{k}$ and $n_{k-1}$ is defined by conditional probability.

In the process of constructing DBN of component, the network size grows proportionally with increment of number of time slices. We reduced the size of network by using the iterative reasoning approach. States of component at different time slice are modeled by a simply DBN including two nodes as presented in Fig. (2).

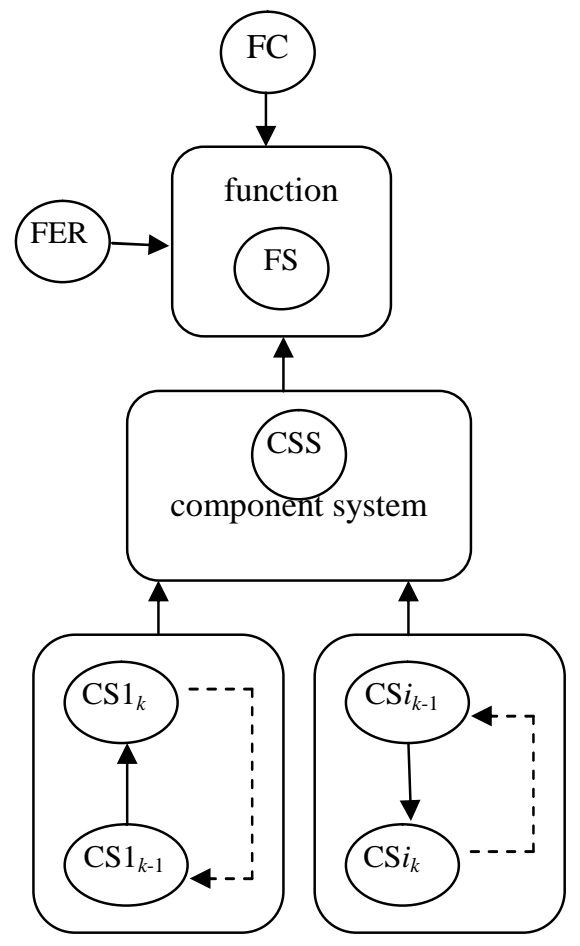

Fig. (2). DBN structure for the component

Fig.3 OODBN for fault diagnosis

The Eq. 1 is used to compute the normal probability of component at time slice $\mathrm{k}$ according to failure rate and the sate at previous time slice.

$$
p\left(C_{k}=\text { Normal }\right)=(1-\lambda \Delta t) p\left(C_{k-1}=\text { Normal }\right)
$$

The state probability of component at time slice $k+1$ is computed by replacing the sate probability at time slice $k-1$ with the probability at time slice $k$.

The posterior conditional probability of common causal nodes com $n$ for all fault states can be calculated as follows.

$$
P\left(\mathrm{com}_{-} n \mid F S\right)=\frac{P\left(\mathrm{com}_{-} n\right) P\left(F S \mid \mathrm{com}_{-} n\right)}{P(F S)}
$$

Where $P\left(F S \mid\right.$ com $\left._{-} n\right)=1$, thus

$$
P\left(\text { com_ }_{-} \mid F S\right)=\frac{P\left(\text { com }_{-} n\right)}{P(F S)}
$$

The function is in a failure state when we diagnose system. Considering $P(F S)$ as a scaling factor, conditional probability $P\left(\mathrm{com}_{-} n \mid F S\right)$ can be determined by $P\left(\mathrm{com}_{-} n\right)$.

The posterior conditional probability of causal node only $n$ belonging to a fault state is computed as follows. 


$$
\begin{aligned}
& P\left(\text { only_}_{-} n \mid F S_{k}\right)=\frac{P\left(\text { only_n }_{-}\right)}{P\left(F S_{k}\right)} \\
& P\left(F S_{k}\right)=P\left(F S_{k} \mid F S\right) P(F S)
\end{aligned}
$$

Where $F S_{k}$ is a definite failure state. Considering $P(F S)$ as a scaling factor, thus,

$$
P\left(\text { only_ }_{-} \mid F S_{k}=\frac{P\left(\text { only_n}_{2}\right)}{P\left(F S_{k} \mid F S\right)}\right.
$$

\section{Diagnosis For ISG-Engine Case}

With the increase in complexity of automobiles, automotive system has more and more components and sub-systems that interact with each other, fault diagnosis are becoming increasingly important and difficult. A diagnosis model that detects and localizes faults is thus needed, both as an aid in the repair process and for detecting and isolating faults. The proposed method is applied to an example of a ISG-engine system. The configuration of ISG-engine hybrid powertrain is shown in Fig. (4) .

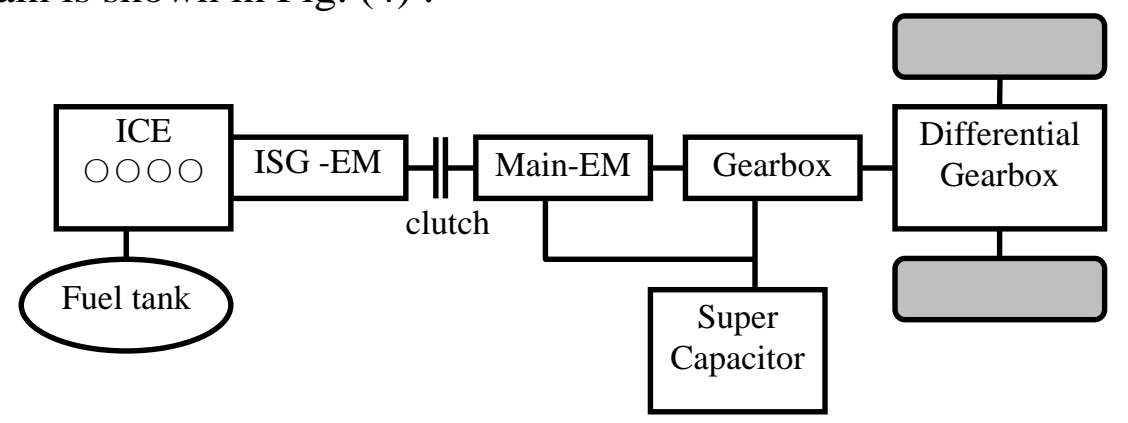

\section{FMEA Of ISG-Engine}

\begin{tabular}{|c|c|c|c|c|c|}
\hline function & cub_cystem & & & effects & \\
\hline \multirow{3}{*}{ Fuel supply } & \multirow{3}{*}{$\begin{array}{l}\text { Fuel supply } \\
\text { system }\end{array}$} & & & & \\
\hline & & Fuel injector & leak & extinction & assembly damaged \\
\hline & & injection pump & Leak, catching & $\begin{array}{c}\text { Insufficient power, } \\
\text { extinction }\end{array}$ & $\begin{array}{l}\text { Plunger matching } \\
\text { parts damaged }\end{array}$ \\
\hline \multirow{2}{*}{ Air supply } & \multirow{2}{*}{ Air supply system } & $\begin{array}{c}\text { Throttle position } \\
\text { sensor }\end{array}$ & $\begin{array}{c}\text { Throttle } \\
\text { opening error }\end{array}$ & Unstable idle speed & Sensor damaged \\
\hline & & Air flow meter & Data error & Insufficient power & Circuit failure \\
\hline \multirow{3}{*}{ firing } & \multirow{3}{*}{ Firing system } & Spark plug & $\begin{array}{c}\text { Electrode melt } \\
\text { etc }\end{array}$ & $\begin{array}{c}\text { Preignition, } \\
\text { Severe vibration }\end{array}$ & Small clearance \\
\hline & & Ignition coil & \begin{tabular}{|c|} 
Short circuit, \\
Broken circuit
\end{tabular} & $\begin{array}{l}\text { Star-up failure, } \\
\text { Unstable idle speed }\end{array}$ & Coil breakage \\
\hline & & subloop & \begin{tabular}{|c|} 
Short circuit, \\
Broken circuit
\end{tabular} & $\begin{array}{c}\text { Star-up failure, } \\
\text { Unstable idle speed }\end{array}$ & Coil breakage \\
\hline \multirow[t]{2}{*}{$\begin{array}{c}\text { Gas } \\
\text { distribution }\end{array}$} & \multirow{2}{*}{ Valve mechanism } & valve & $\begin{array}{c}\text { Abnormal } \\
\text { sound }\end{array}$ & Supply shortage & Excessive clearance \\
\hline & & gear & wear & Impact, vibration & Gear precision \\
\hline \multirow{4}{*}{ Power export } & \multirow{4}{*}{$\begin{array}{l}\text { Crank-link } \\
\text { mechanism }\end{array}$} & crankshaft & crack & Unstable operation & Fatigue stress \\
\hline & & Piston, piston ring & Crack, wear & Insufficient power & friction \\
\hline & & cylinder & Crack, wear & Insufficient power & friction \\
\hline & & Cylinder head & crack & Insufficient power & Temperature changes \\
\hline \multirow[t]{2}{*}{ starting } & \multirow[t]{2}{*}{ ISG-EM } & Stator & Crack, wear & Function failure & $\begin{array}{c}\text { Support structure } \\
\text { relaxation }\end{array}$ \\
\hline & & rotor & Fall off & Rotor stuck & vibration \\
\hline
\end{tabular}

Fig. (4) Configuration of a hybrid powertrain

Table 1 parts FMEA of ISG-engine 
The components of ISG-engine are indexed in the FMEA. In Table 1, parts FMEA of ISG-engine are given. The failure mode of each element is defined as well as its effect. The cause is linked with the element states.

\section{OODBN Model Of ISG-Engine}

Fig. (5) shows a OODBN for power export function of ISG-engine based on structure-function and failure mode effect analysis. The diagnostic model consists of four classes, i.e. function class, component system class, component class and observation class. The main function of engine is power export. Thus, 'power export' is the highest level of OODBN. The function states are normal, insufficient power, high idle speed, low idle speed, extinction, high fuel consumption. FER affecting 'power export' are 'gas distribution function', 'fuel supply function' and the 'air supply function'.

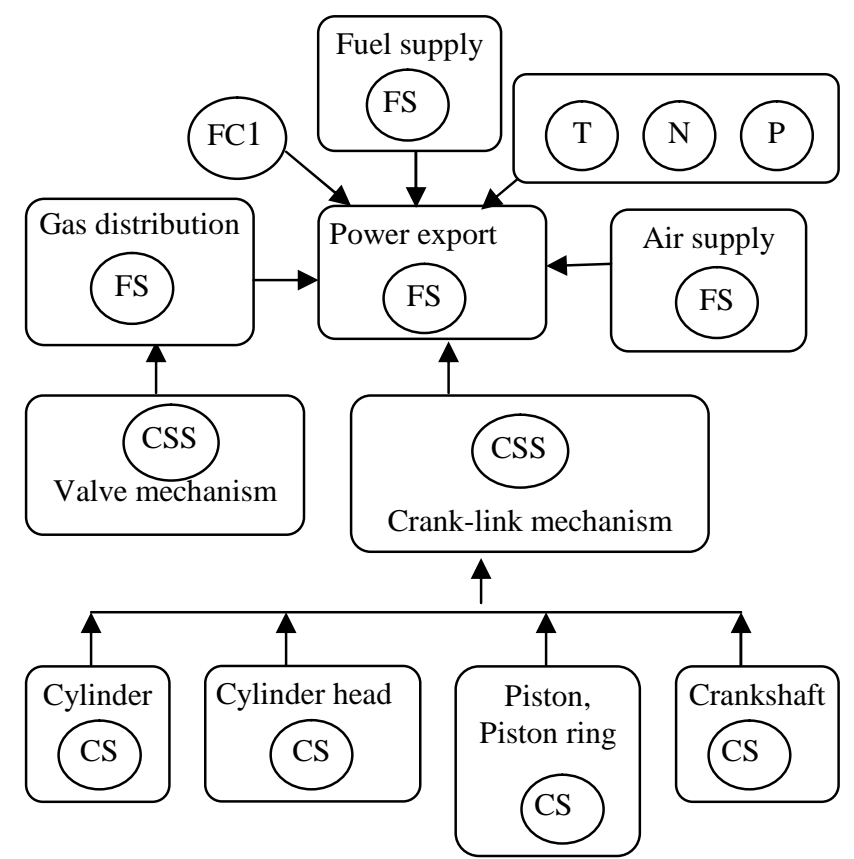

Fig. (5) OODBN for power expert function

The observation node consists of torque $T$, revolutions per minute $N$, power $P$. The Mean Time to Failure (MTTF)of each component can be extracted from historic fault data of hybrid electric buses. Then we reason the dynamic fault probability using an iterative procedure realized

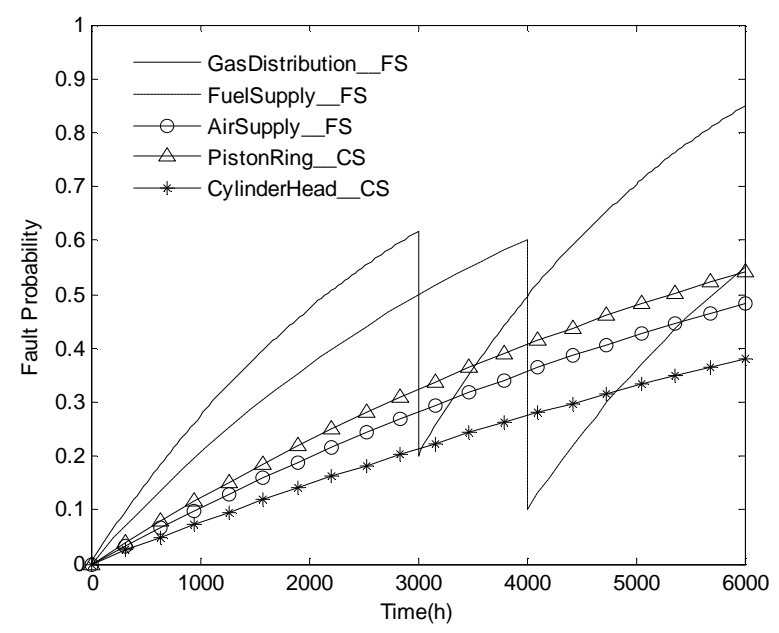

Fig. (6) Fault probability of main nodes for insufficient power state

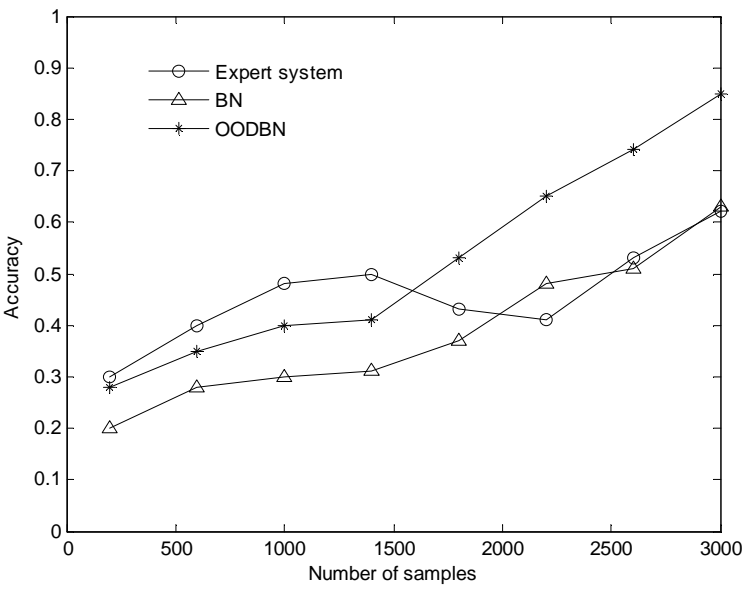

Fig. (7) Accuracy comparison of three methods 
Fig. (6) shows the fault probabilities of main nodes for individual function state 'Insufficient power' according to the time step.

From Fig. (6), we can find out that when the function sate of 'power export' is 'insufficient power', the function nodes powerfully influencing on the function are 'gas distribution', 'fuel supply', the component nodes are 'Piston ring' and 'Cylinder head' successively in 3000 hours ago. Referring to fault probability and complexity of nodes, the diagnostic order is 'Piston ring', 'Cylinder head', 'valve mechanism', 'fuel supply system', 'air supply system'. According to above method, we construct OODBN for sub-function 'Gas distribution' and depict dynamic fault probability of relevant nodes. In Fig. (6), valve mechanism was repaired, but the normal state probability of node didn't return to 1 , the reason is function degradation of other components.

Expert system is currently the most commonly used method in diagnosis of engine. In order to express the superiority of OODBN, we used two methods (BN, OODBN) to diagnose the same fault. Referring to a expert system of engine, the results are shown in Fig. (7).

Because the OODBN diagnosis model takes into account FMEA and dynamic fault probability of nodes, it has higher accuracy than BN diagnosis model. Since expert system is constructed according to experience of experts, when small number of sample are used, OODBN diagnosis model has lower accuracy, compared with expert system. But with the number of samples increasing, the accuracy of OODBN model is higher and higher. Additionally, expert system makes an accurate judgment difficultly, when there is only a relatively small difference between sensitive parameter and the normal value. OODBN diagnosis model can attain a better diagnosis.

\section{Conclusions}

This paper proposes a new approach of fault diagnosis using OODBN for a complex electromechanical system. We construct the diagnosis model based on function and fault modes analysis in terms of transmission of function flow. The methodology reduces the complexity of model and represents the transmission relationships among function. So the model provides a convenient way for analyzing fault propagation and relevant failure isolation. In particular, the model is hierarchical and dynamical, to depict actual character of nodes. Furthermore, the model are reusable and expandable, it can be expanded to hybrid power-train system.

\section{Acknowledgements}

This work was financially supported by the Transformation of Major Scientific and Technological Achievements of China (2012258).

\section{References}

[1] Wang Yuan-hang, Deng Chao, Wu Jun, et al. Mixed-expert-system-based fault diagnosis for heavy machine[J]. Computer Integrated Manufacturing Systems, 2010(10):2139-2147.

[2] David Leung, Jose Romagnoli. Dynamic probabilistic model-based expert system for fault diagnosis[J]. Computers and Chemical Engineering 24 (2000): 2473-2492

[3] Li Xiang-gang. Application of Bayesian networking in fault diagnosis of car [J]. Highways \&Automotive Applications, 2006, (113):21-24. 
[4] A. Bobbioa, L. Portinalea, M. Minichinob, E. Ciancamerlab. Improving the analysis of dependable systems by mapping fault trees into Bayesian networks[J]. Reliability Engineering and System Safety 71 (2001):249-260

[5] Uri Lerner, Ronald Parr, Daphne Koller. Bayesian Fault Detection and Diagnosis in Dynamic Systems. Proceedings of the Seventeenth National Conference on Artificial Intelligence (AAAI-00), Austin, Texas, August 2000: 531-537

[6] Rongxing DUAN, Jiliang TU, Decun DONG. A Novel Hybrid Approach of Fault Tree and Bayesian Networks for Fault Diagnosis[J]. Journal of Computational Information Systems ,2010(11): 3605-3612

[7] Yoshinobu Kawahara, Takehisa Yairi, and Kazuo Machida. Diagnosis Method for Spacecraft Using Dynamic Bayesian Networks. The 8th International Symposium on Artifical Intelligence, Robotics and Automation in Space-iSAIRAS', Munich, Germany, 2005:5-8

[8] David C Y, Thanh C N, Peter H. Bayesian network model for reliability assessment of power systems[J].IEEE Trans. on Power Systems, 1999,14(2): 426-432.

[9] Bangso O, Wuillemin P-H. Top-down construction and repetitive structures representation in Bayesian Networks. Thirteenth international Florida artificial intelligence research symposium conference, Florida, USA, 2000:282-286

[10] Daphne Koller, Avi Pfeffer. Object-Oriented Bayesian Networks. Proceedings of the Thirteenth Annual Conference on Uncertainty in Artificial Intelligence (UAI-97),Providence, Rhode Island, August 1-3, 1997: 302-313

[11] Weber P, Suhner M.C. An application of Bayesian networks to the performance analysis of a process. Proceedings of lm 13, ESREL 2002 European conference. Lyon, France; 2002.

[12] Shahan Yang,Yuchen Zhou, John Baras. Compositional Analysis of Dynamic Bayesian Networks and Applications to Complex Dynamic System Decomposition[J]. Procedia Computer Science, 2013(16): 167-176 